\title{
La vinculación con la sociedad como eje multidisciplinario en el desarrollo de capacidades locales en turismo y la hospitalidad. Caso de estudio: Parroquia de Cangahua
}

\section{Linkage with society as a multidisciplinary main point in the local development of tourism and hospitality capabilities. Case Study: Parish of Cangahua}

\author{
Miguel Yuctor Álvarez ${ }^{1 *}$, Luis Benalcázar Carvajal ${ }^{1}$, Daniel Fierro Mosquera ${ }^{1}$ y Gabriela Moncayo Silva ${ }^{1}$ \\ ${ }^{1}$ Universidad UTE \\ *miguel.yuctor@ute.edu.ec
}

DOI: https://doi.org/10.26871/killkana_social.v3i2.463

\begin{abstract}
Resumen
En la parroquia de Cangahua, existe un turismo incipiente. Los factores incidentes en este problema son el desconocimiento del potencial turístico del territorio, los productos y servicios deficientes, la concentración de actividades económicas en agricultura y ganadería, y la poca gestión de instituciones públicas inherentes al territorio. Esta situación tiene como efectos la escasa diversificación de alternativas productivas y económicas, la subutilización de recursos, el poco aprovechamiento de los atractivos naturales y culturales, así como el escaso posicionamiento de los emprendimientos turísticos, hoteleros y gastronómicos. Como una alternativa de solución a este problema, la Universidad (“UTE”) presentó el proyecto de vinculación con la sociedad "Propuesta de desarrollo turístico sostenible en la parroquia de Cangahua", en el que la Dirección de Vinculación con la Sociedad y las Facultades de Hospitalidad y Servicios, Comunicación, Artes y Humanidades, y Arquitectura y Urbanismo se encuentran trabajando con miras al desarrollo local. En tal sentido, la presente investigación, hace referencia a aspectos del proyecto que tienen que ver con el desarrollo integral de la comunidad, el mejoramiento de la calidad de vida y la participación comunitaria e institucional dentro del desarrollo de capacidades locales.
\end{abstract}

Palabras clave: vinculación con la sociedad, turismo y hospitalidad, multidisciplinario.

\begin{abstract}
The parish of Cangahua shows an incipient tourism development. The aspects influecing this problem are the lack of knowledge on this area's tourism potential, The poor quality of its products and services, the fact that economic activities are mainly concentrated in agriculture and livestock farming; and the deficient management of the area's public institutions. As a consequences of this situation, there is limited variation on productive and economic alternatives, poor exploitation of natural resources and cultural attractions, as well as the lack of tourism, hotel and gastronomic entrepreneurships. As an alternative solution to this problem, the University ("UTE") introduced the linkage with the society project, titled "Proposal for sustainable tourism development in the parish of Cangahua". The Linkage with the Society Directorate as well as the faculties of Hospitality and Services, Communication, Arts and Humanities, and Architecture and Urbanism, are working towards the local development of this parish. With reference to this matter, the present research refers to those aspects of the project that deal with the community's integral development, the quality of life improvement, as well as the community's and institutional participation, as part of the local capabilities development.
\end{abstract}

Key words: linkage with society, tourism and hospitality, multidisciplinary.

\section{Introducción}

La Educación Superior en Ecuador se ha transformado en los últimos años y ha generado varios lineamientos que se basan en los principios constitucionales para enfocarse en leyes orgánicas y sus respectivos reglamentos, los cuales consideran funciones sustantivas como docencia, investigación y vinculación. Esta última trata de cambiar los factores sociales de los sectores y/o territorios vulnerables que de acuerdo a cada realidad institucional, prioriza acciones y designa el talento humano y los recursos necesarios para 
la movilización de la comunidad universitaria: docentes y estudiantes.

De igual forma, los programas de vinculación con la sociedad de las Instituciones de Educación superior (IES), deben estar alineados hacia el cumplimiento de directrices que parten desde dos enfoques: el primero, el público, que abarca desde el Plan Nacional de Desarrollo, agendas zonales, planes de desarrollo y ordenamiento territorial, entre otros; y segundo desde el enfoque institucional, es decir, desde lo estipulado por las IES en su Plan Estratégico de Desarrollo Institucional, lo que evidencia una articulación de los esfuerzos, para el desarrollo local de los territorios.

El cantón Cayambe, ubicado en la provincia de Pichincha, está conformado por dos parroquias urbanas: Juan Montalvo y Cayambe, y seis parroquias rurales: Ascázubi, Cangahua, Olmedo, Otón, San José de Ayora y Santa Rosa de Cusubamba.

Cangahua es una de las parroquias rurales más antiguas del cantón Cayambe y está situada en el sureste del cantón. Fue fundada el 29 de octubre de 1790. Posee una superficie de $332,35 \mathrm{~km} 2$. Tiene una población de 16231 habitantes, con 7920 hombres y 8311 mujeres. De acuerdo a la información del censo 2010, Cangahua tiene una población con nacionalidad prevalente: kayambi, kichwa de la Sierra, kitukara, Otavalo y Salasaka; en la parroquia no se ha establecido una organización y/o asentamientos definidos de población por nacionalidad. 7384 personas (45\%) hablan el idioma kichwa (Gobierno Autónomo Descentralizado de Cangahua, 2012)

La población económicamente activa de la parroquia Cangahua se encuentra ocupada en su mayor parte en actividades de agricultura, ganadería, silvicultura y pesca con $63,0 \%$; a la construcción el $10 \%$; comercio al por mayor y menor el $3 \%$, y a las industrias manufactureras el $2 \%$. Los grupos de ocupación en la parroquia se conforman por agricultores y trabajadores en el $45 \%$, el $22 \%$ ocupaciones elementales, oficiales, operarios y artesanos el 9\%; la mayoría de actividades son consideradas como familiares y no como microempresas (Gobierno Autónomo Descentralizado de Cangahua, 2012).

El $85 \%$ de la población tiene acceso a energía eléctrica, pero se carece de alumbrado público, excepto en el centro poblado. La recolección de basura se realiza semanalmente en la zona urbana y en el $4 \%$ de las comunas del sector rural; en las otras comunidades, se procede a quemar o enterrar la basura. En varias comunidades de la parroquia, existen dos subcentros de salud y cuatro dispensarios del Seguro Campesino (Gobierno Autónomo Descentralizado de Cangahua, 2012)

La falta de empleo y las pocas oportunidades de estudio originaron un proceso migratorio dentro y fuera del país. Las personas que han migrado hacia el exterior son aproximadamente 83 personas, las que se han movilizado en especial a España, Estados Unidos y Cuba (Instituto Nacional de Estadísticas y Censos, 2010).
A partir de lo antes expuesto, se identificó que la población se dedica solo a subsistir mediante las actividades agrícolas y ganaderas sin aprovechar sus recursos culturales y naturales. Las causas de este problema son el desconocimiento del potencial turístico, los productos y servicios turísticos deficientes, concentración de actividades económicas en agricultura y ganadería y poca gestión de los gobiernos autónomos descentralizados. En consecuencia, este problema genera poca actividad turística, turismo incipiente, bajo aprovechamiento de los recursos turísticos naturales y culturales, así como de los emprendimientos de turismo y hospitalidad.

Esta situación conlleva a que los ingresos familiares se encuentren limitados.

La mayoría de la población de la parroquia está dedicada a actividades agrícolas, ganaderas y avícolas, de las cuales en su gran mayoría son consideradas como familiares y no como microempresas. Apenas un $1 \%$ de la población de la parroquia de Cangahua se dedica a actividades turísticas y de servicios (GAD Parroquial Cangahua, 2012). Con estos datos se estructuró la línea base del proyecto.

Sobre la información proporcionada en la línea base indicada, el proyecto intervino en el $1 \%$ de la población de la parroquia de Cangahua que se dedica a actividades turísticas y de servicios. Por motivo, de que los habitantes de Cangahua han visto limitados sus ingresos debido a que estos van en función de sus extensiones de terreno y la concentración de actividades económicas como las agrícolas y la construcción, la principal problemática encontrada es pobreza y falta de diversificación en las actividades económicas de la zona.

Con base en las consideraciones anteriores, el proyecto pretendió sensibilizar a la comunidad en la diversificación de actividades económicas y el rescate de los atractivos naturales y culturales de la parroquia de Cangahua y sobre todo a que los pobladores no solo sean considerados como objetos sino actores dentro de los procesos de desarrollo local a través de la gestión del turismo y la hospitalidad.

\section{Marco teórico}

Para el presente estudio se determinaron varias definiciones que se encuentran discutiendo dentro del contexto ecuatoriano sobre el tema de vinculación. Sin embargo, el marco teórico parte desde la pertinencia de la oferta académica de las IES, más lo que se encuentra establecido dentro de la Ley Orgánica de Educación Superior, (LOES) y el Reglamento de Régimen Académico sobre la importancia de la vinculación con la sociedad para el desarrollo de los territorios.

Desde el principio de pertinencia se menciona que "la educación superior responda a las expectativas y necesidades de la sociedad a la planificación nacional y al régimen de desarrollo [...]. Para ello, las instituciones de educación superior articularán su oferta docente, de investigación y actividades de vinculación con la sociedad" (Asamblea Nacional del Ecuador, 2011). Lo que sustenta 
que, desde la normativa vigente, vinculación es importante para que los territorios cambien sus condiciones a través del intercambio y construcción de conocimientos que permitan el mejoramiento de la calidad de vida de los pobladores.

En ese sentido, la Universidad UTE dispone que "vinculación con la sociedad hace referencia a los programas de educación continua, gestión de redes, cooperación y desarrollo, relaciones internacionales, difusión y distribución del saber que permitan la democratización del conocimiento y el desarrollo de la innovación social" (Universidad UTE, 2017)

De igual forma se menciona que vinculación es

[..] el modelo se afianza en propuestas comunitarias acorde a los cambios sociales que vive nuestra región. Por ende, amerita un diagnóstico situacional que refleje las etapas de asentamiento poblacional, su visión y esperanza de mejores días. Partiendo de esta realidad, se promueve la participación educativa para mejorar la calidad de vida de los habitantes. El compromiso universitario se fundamenta en una estrategia motivacional hacia los estudiantes para que conozcan la realidad social de su entorno, lo que se logra haciendo que ellos participen en todas las instancias de un plan de vinculación con la comunidad. (Orozco y Andrea, 2011)

De igual forma, "es una de las funciones sustantivas de la educación superior que, de conjunto con la formación académica y la investigación, tiene el propósito de solucionar problemas de la comunidad, a través de proyectos, prácticas pre-profesionales, capacitación y otros servicios profesionales, que posibilitan la comunicación y la interacción efectiva de la universidad con su entorno, así como la integración de la teoría con la práctica del quehacer universitario en función del desarrollo social sostenible" (Polaino y Antonio, 2017). Con relación a lo anterior, Toledo, et, al (2017), menciona que vinculación es "la función que cumple la universidad con el medio externo. Se apoya en la docencia y la investigación para el diseño y desarrollo de proyectos que articulen los conocimientos científicos y técnicos con los saberes y experiencias del entorno, para ello se requiere de una política cultural coherente en un territorio, en una ciudad o en un país". (De Aparicio, Chininin Macanchi, y Toledo Rodríguez, 2017)

Finalmente, se puede considerar a vinculación con la sociedad como "interacción de la universidad con los demás componentes de la sociedad, para mutuo beneficio en el avance del conocimiento, formación de recursos humanos, solución de problemas específicos y respuesta a las situaciones de pobreza del país" (Saltos Briones, Odriozola Guitrat, y Ortiz Torres, 2018)

\section{Metodología}

La metodología para la intervención en el territorio desde la Universidad UTE y desde la Unidad de Vinculación con la Sociedad parte desde dos enfoques:
Primero: Institucional. La Dirección de Vinculación trabaja desde un lineamiento integrador de conocimientos, es decir, la intervención de las distintas unidades académicas dentro del territorio priorizado bajo el siguiente modelo de gestión (Ver anexo 1):

- Intervención en territorios priorizados, en donde busca la aplicación del conocimiento pertinente de las carreras que se imparten a nivel nacional en las diferentes unidades académicas para partir de las necesidades de la población del territorio priorizado mediante el análisis de la vulnerabilidad social, y plantear soluciones viables orientadas al fomento del desarrollo local.

- Programas de vinculación que constituyen el nexo que posibilita la relación directa y permanente entre la Universidad y su entorno, y entre aquella y las realidades productivas y sociales. Comprende toda la oferta de proyectos inherentes a la formación académica y humanista que se imparte en la UTE. Los programas se enmarcan dentro del área de intervención de territorios priorizados y deberán formularse de acuerdo al perfil de egreso de cada carrera, respondiendo a las áreas, subáreas y áreas específicas del conocimiento.

- Proyectos multidisciplinarios que se ejecutan en territorios priorizados en un marco de acción de principios, valores y responsabilidad social para la aplicación del conocimiento. Están alineados a los planes de desarrollo y ordenamiento territorial y consolidan su cumplimiento con alianzas estratégicas con actores externos públicos y privados establecidos mediante convenios de cooperación (Universidad UTE, 2017).

Segundo: Desarrollo del proyecto en el que, en el caso de estudio, se utilizaron las siguientes metodologías por cada componente del proyecto:

Componente 1. Diagnóstico del sistema turístico de la parroquia Cangahua

Se realizó el levantamiento de la información para el diagnóstico del sistema turístico con las siguientes metodologías:

- Metodología de inventario y jerarquización de atractivos turísticos del Ministerio de Turismo (MINTUR). Participaron un técnico facilitador del MINTUR, docentes y estudiantes de la Facultad de Hospitalidad y Servicios.

- Metodología para el levantamiento del catastro de alimentos, bebidas y alojamiento, facilitado por parte del equipo técnico especializado de la Facultad de Hospitalidad y Servicios, docentes y estudiantes.

Estas metodologías fueron diseñadas por los organismos de control como el Ministerio de Turismo del Ecuador y Quito Turismo-UTE. Así mismo, se identificaron en forma transversal las actividades económicas, actores, indicadores sociales y asentamientos humanos.

Como parte del diagnóstico, se realizó el levantamiento de la información de fuentes secundarias: publicaciones, tesis, planes de manejo, mapas, planes de desarrollo provincial, cantonal y parroquial, por motivo de que la visita 
in situ aportó con una visión integral del territorio y de la información que se debe recopilar para un diagnóstico eficiente y eficaz; para lo cual se ejecutaron talleres de capacitación a estudiantes y docentes sobre las metodologías que se aplicaron en territorio.

Luego, se efectuó la visita técnica de campo, a fin de que los docentes y estudiantes se familiaricen de forma preliminar con el territorio de estudio. Además se establecieron los contactos iniciales con las entidades cooperantes y representantes de las comunidades para facilitar la intervención en el territorio.

Se inventariaron y jerarquizaron los atractivos turísticos de la parroquia de Cangahua; así como también, mediante brigadas de trabajo por carrera se procedió con la identificación y categorización de la oferta gastronómica y hotelera del área de intervención del proyecto.

Los resultados del inventario y jerarquización de atractivos turísticos y del catastro de alojamiento y gastronómico de la parroquia de Cangahua fueron validados con la comunidad a través de un taller de trabajo, luego de lo cual se realizaron los ajustes al documento preliminar y se elaboró el informe del diagnóstico del sistema turístico de la parroquia de Cangahua.

Componente 2. Propuesta de intervención turística con base en potencialidades identificadas en la fase de diagnóstico para el aprovechamiento de los recursos turísticos de la parroquia de Cangahua

La propuesta de intervención turística para el aprovechamiento sostenible de los recursos turísticos de la parroquia de Cangahua, con la participación de estudiantes y docentes de las carreras: Administración de Empresas Turísticas y Conservación Ambiental, Administración Hotelera y Gastronomía, aportó técnicamente en los siguientes aspectos:

a) Definición del modelo de la propuesta.

b) Directrices generales hacia el equipo de trabajo.

c) Zonificación del territorio.

d) Estrategias turísticas de intervención.

Tomando como base las potencialidades turísticas identificadas en la fase de diagnóstico, las preferencias de la demanda y el interés de la población local, se procedió con el desarrollo de la propuesta de intervención, la que incluyó la misión, visión, objetivos, programas, estrategias y acciones para un desarrollo turístico sostenible, para lo cual se ejecutaron talleres participativos con la población local para la construcción de la propuesta.

Con relación al desarrollo de la propuesta, se definieron las zonas de intervención con base en las características, uso actual y potencial que se generan en el sector. Se elaboró el mapa de zonificación turística de la parroquia (zonas, áreas y/o centros), y se lo socializó en un taller con la comunidad.

A partir del análisis que realizaron los actores involucrados se concretaron estrategias con base en las potencialidades del territorio; por tanto, se estructuró la oferta turística, hotelera y gastronómica de acuerdo al potencial existente en la zona. Esto comprende los bienes y servicios, recursos e infraestructura ordenados de forma que sean ofertados al mercado para ser consumidos por el visitante o turista.

Componente 3. Socialización y gestión de la propuesta de intervención turística y generación de la imagen y promoción turística de la parroquia

Se desarrolló un proceso de gestión de la propuesta de intervención, a través de sensibilización de la población, modelos organizativos, comercialización y alianzas estratégicas, por parte de los estudiantes y docentes de la Facultad.

La socialización de la propuesta de intervención turística permitió difundir, en la comunidad de Cangahua, las acciones concretas que fueron implementadas a través de talleres con la participación de la comunidad. A partir del proceso de socialización, se realizó el levantamiento de actores claves relacionados con la gestión turística en la parroquia de Cangahua, en el área de influencia con la finalidad de motivar a la comunidad de Cangahua sobre las oportunidades que ofrecen las actividades turísticas, hoteleras y gastronómicas en la generación de ingresos económicos alternativos mediante la conformación de grupos de emprendedores y acuerdos con las asociaciones enfocadas en las áreas de hospitalidad y servicios.

Para el desarrollo de la imagen de los emprendimientos intervino la Facultad de Comunicación, Artes y Humanidades que estableció los lineamientos en los que se basaron en el uso de software, utilización de medios visuales para la promoción turística.

Componente 4. Capacitación a la población local acorde a la planificación de intervención en el área de hospitalidad y servicios y se han diseñado propuestas de emprendimientos productivos en el área de turismo, hotelería y gastronomía.

Se implementaron las acciones priorizadas de la propuesta de intervención, dando énfasis en la capacitación a la población local en temas turísticos, hoteleros y gastronómicos con el fin de que los actores locales adquieran conocimientos técnicos y desarrollen competencias laborales. Además, se trabajó en el desarrollo de productos promisorios que permitan la innovación turística sostenible con participación local.

Durante la ejecución de este componente, se realizó el acompañamiento a los participantes para la implementación de las propuestas de intervención y la contraparte técnica de los GAD para garantizar la sostenibilidad de las propuestas.

Se ejecutaron capacitaciones de acuerdo a las necesidades de la parroquia en cooperación con los involucrados que incluyó prácticas demostrativas, talleres programados, con insumos y material audiovisual para el desarrollo de nuevas competencias laborales

Se desarrollaron productos promisorios con base en las potencialidades identificadas, la demanda, el interés de la comunidad local y facilidades de acceso; así mismo, se definieron estrategias preliminares para su comercialización. 


\section{Resultados}

Los resultados se basan en los productos entregables de cada uno de los componentes que se detallaron en la metodología, por lo que a continuación se describe un consolidado de estos:

\section{Componente 1:}

Por motivo de que el componente 1 abarcó el diagnóstico territorial y turístico de la parroquia de Cangahua, se pueden resaltar los siguientes resultados:

Población objetivo programada: Beneficiarios directos:

Tabla 1 Población objetivo

\begin{tabular}{|c|c|c|}
\hline Hombres & Mujeres & Total en número \\
\hline 94 & 101 & 195 \\
\hline
\end{tabular}

Fuente: Universidad UTE- Proyecto Vinculación Cangahua

Las beneficiarias directas son 195 personas, de las cuales son 94 hombres (48\%) y 101 mujeres (52\%), lo que comprueba que es un territorio priorizado, debido a la fuerte migración que se suscita desde el campo hacia la ciudad. Así mismo, muestra que la participación de la mujer es importante dentro de los procesos productivos y aún más su consolidación de los emprendimientos turísticos a través de la gestión.

Tabla 2 Población atendida por asociación

\begin{tabular}{|c|c|c|c|c|c|c|}
\hline \multirow{2}{*}{ Ítem } & \multirow{2}{*}{ Nombre } & Hombres & Mujeres & $\begin{array}{c}\text { Con } \\
\text { disca- } \\
\text { pacidad }\end{array}$ & $\begin{array}{c}\text { Sin } \\
\text { disca- } \\
\text { pacidad }\end{array}$ & $\begin{array}{c}\text { Total en } \\
\text { número }\end{array}$ \\
\cline { 3 - 7 } & Asoturcay & 10 & 11 & & 0 & 21 \\
\hline 2 & $\begin{array}{c}\text { Chumillos } \\
\text { Central }\end{array}$ & 37 & 38 & & 0 & 75 \\
\hline 3 & $\begin{array}{c}\text { Chumillos } \\
\text { Alto }\end{array}$ & 30 & 32 & & 0 & 62 \\
\hline 4 & $\begin{array}{c}\text { Asociación } \\
\text { El } \\
\text { Vicundo }\end{array}$ & 11 & 12 & & 0 & 23 \\
\hline 5 & $\begin{array}{c}\text { Jorafanga } \\
\text { (Cunibu- } \\
\text { ro) }\end{array}$ & 6 & 8 & & 0 & 14 \\
\hline & \multicolumn{1}{|l|}{} & & & & Totales & 195 \\
\hline
\end{tabular}

Fuente: Universidad UTE-Proyecto de Vinculación con la Sociedad parroquia de Cangahua

La asociación juega un papel preponderante dentro del desarrollo local. Por tal motivo, el proyecto trató de involucrar a la mayor cantidad de asociaciones con sus integrantes: Chumillos Central con 75 miembros, Chumillos Alto con 62, ASOTURCAY con 21, Asociación El Vicundo con 23 y JORAFANGA con 14. Cabe mencionar que, en todo el proceso de diagnóstico se identificaron a las asociaciones que ya tenían un producto turístico en iniciación, esto debido a que dentro del componente 4 existen proyectos de mejoramiento y sostenibilidad de los emprendimientos en el área de turismo, hotelería y gastronomía.

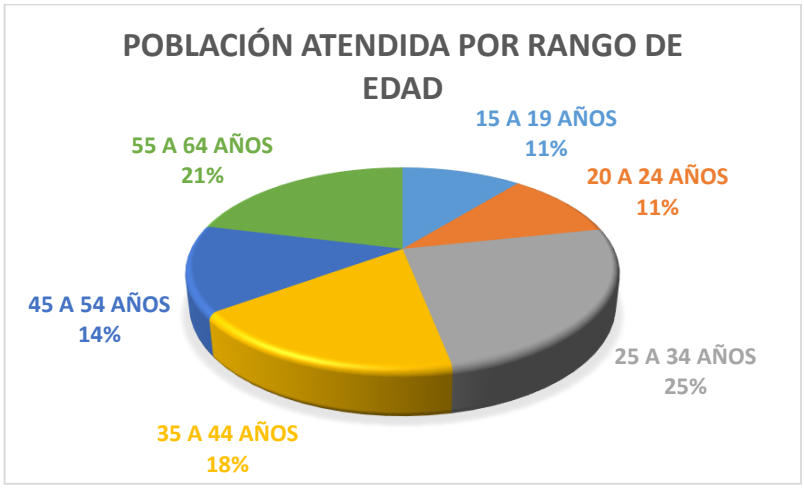

Figura 1. Población atendida por rango de edad

La población atendida por rango de edad es representada por jóvenes de 25 a 34 años que equivale a los $25 \%$, seguidos de personas de 55 a 64 años con un $21 \%$ y finalmente de 34 a 44 años con un $18 \%$. Lo que evidencia que la gente joven está consciente del potencial turístico que tiene el territorio y está iniciando sus emprendimientos en turismo y hospitalidad y se encuentran apoyados por el grupo etario de mayor edad. 55-64 años (21\%). Por lo cual la participación de la universidad es primordial en el desarrollo de capacidades locales para la comprensión e implementación de herramientas que mejoren los emprendimientos y/o generen nuevas ideas de negocio.

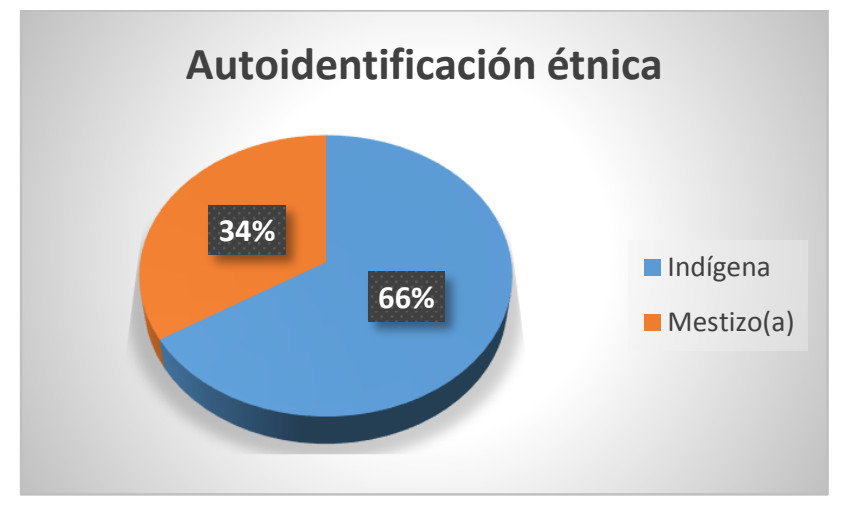

Figura 2. Autoidentificación étnica

Se puede evidenciar y comprobar (figura 2), con las visitas de campo y el levantamiento de información realizados en el diagnóstico que Cangahua es un territorio poblado por indígenas. En el proyecto, el $66 \%$ de los participantes se reconoce como indígena, ya que en Cangahua la población en su mayoría se dedica a actividades agrícolas y ganaderas, por tal motivo, el desarrollo de capacidades locales del proyecto tuvo un enfoque rural adaptándose a las condiciones del entorno.

Dentro de los ámbitos del turismo y de la hospitalidad, se obtuvieron los siguientes resultados: Desde el enfoque de 
materia prima del turismo, es decir, sus recursos ${ }^{1}$ y atractivos $^{2}$, el territorio de Cangahua cuenta con los siguientes componentes del sistema turístico:

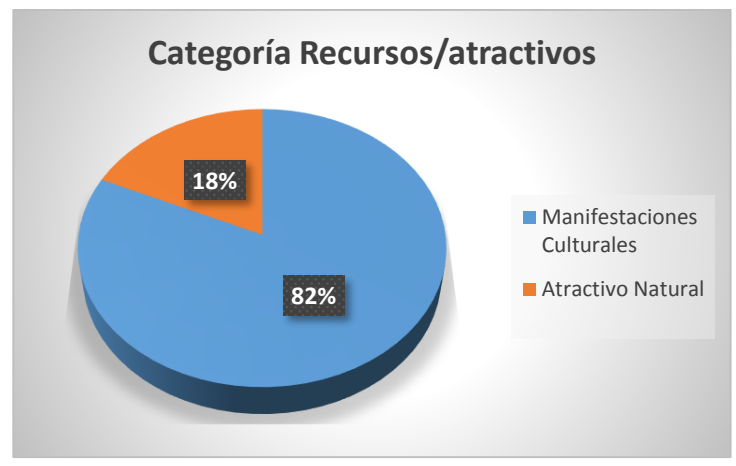

Figura 3. Autoidentificación étnica

Los atractivos que posee Cangahua corresponden a atractivos naturales con un $18 \%$ y a manifestaciones culturales con un $82 \%$. Los productos turísticos que se han creado se enfocan hacia la parte natural, con valor agregado de la cultura indígena.

Tabla 3 Sector de la hospitalidad

\begin{tabular}{|c|c|}
\hline Sector & $\mathrm{N}^{\circ}$ establecimientos \\
\hline Alojamiento & 3 \\
\hline Alimentos y bebidas & 17 \\
\hline
\end{tabular}

Fuente: Universidad UTE-Proyecto de vinculación con la Sociedad parroquia de Cangahua

Dentro del sector de alojamiento, en el territorio se identificaron tres servicios -hostales y área de camping-. Los establecimientos de alimentos y bebidas suman un total de 17, en categorías como restaurantes, soda bares, entre otros.

Por lo expuesto, se hace evidente que Cangahua está en un proceso de desarrollo e implementación de la planta turística necesaria para la satisfacción de las necesidades del turista que se moviliza hacia el territorio.

\section{Componente 2}

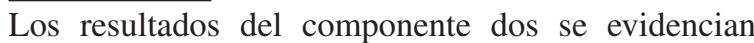
desde el ámbito visual, debido a que se elaboraron mapas temáticos. Uno, en donde se encuentran identificados geográficamente los servicios y atractivos turísticos para dimensionar el alcance de la oferta del territorio. Otro, en donde se coloca la zonificación turística y se determina lo que es zona, área y centro turístico para la atracción de la demanda -turistas-.

\footnotetext{
${ }^{1}$ Recurso. Materia prima del turismo, no tiene aún accesibilidad, señalización ni infraestructura, no se encuentra dentro de la oferta turística.

${ }^{2}$ Atractivo, natural o cultural que motiva al turista para su vista, consta de la infraestructura adecuada, para el disfrute y se encuentra dentro del mercado turístico.
}

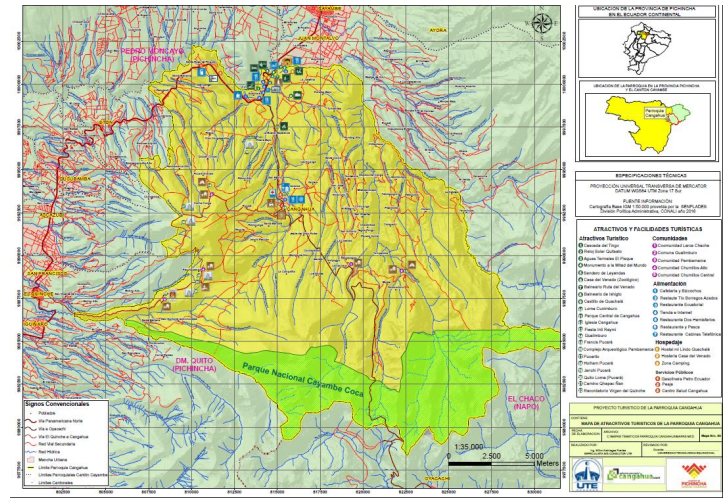

Figura 4. Mapa turístico de Cangahua

Fuente: Universidad UTE-Proyecto de vinculación con la Sociedad parroquia de Cangahua

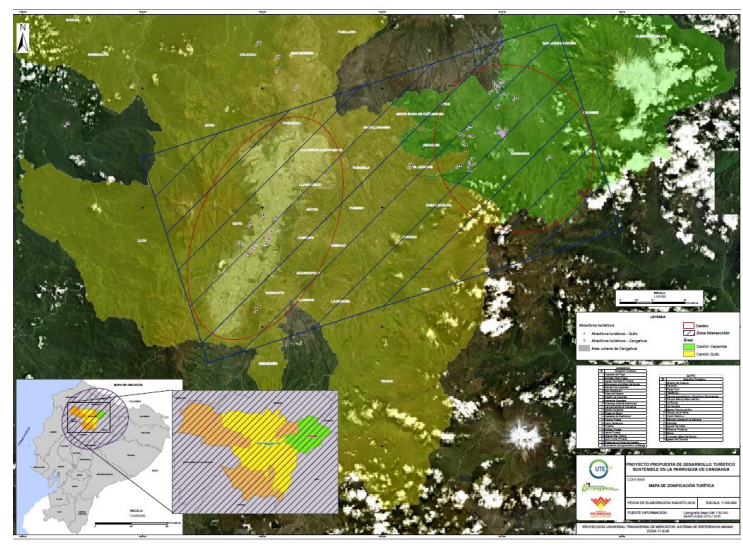

Figura 5. Mapa de Zonificación turística

Fuente: Universidad UTE-Proyecto de vinculación con la Sociedad parroquia de Cangahua

La ilustración muestra la definición de una zona y dos áreas interrelacionadas, en consideración con que el flujo de visitantes que llega a Cangahua proviene de Quito. El área de Cangahua encierra a todos los atractivos identificados en el territorio, luego del diagnóstico realizado por el equipo de vinculación de la Universidad UTE. Cabe mencionar, además, que la concentración de atractivos en la parroquia ha permitido conformar centralidades turísticas con el fin de elaborar productos turísticos determinados que conecten dichos atractivos, este proceso está basado en la distribución espacial definida por la ley de continuidad (Boullon,1997). Esto se puede evidenciar dentro de los productos turísticos que se elaboraron.

\section{Componente 3}

$\overline{\text { El componente }} 3$ son los productos que de acuerdo a la participación activa de las personas, se logró planificar para su posterior implementación en el territorio. Aquí se puede encontrar un plan de desarrollo turístico que cuenta con los lineamientos necesarios para el desarrollo turístico de la parroquia. Consta de 4 rutas turísticas con la participación 
de la comunidad y un formato de organigramas para el desarrollo de funciones que permitan la operación y gestión turística:

Tabla 4 Productos componente 3

\begin{tabular}{|l|l|}
\hline Producto & Detalle \\
\hline $\begin{array}{l}\text { Plan de Desarrollo turís- } \\
\text { tico }\end{array}$ & 1 Plan de desarrollo turístico \\
\hline Rutas turísticas & 4 rutas turísticas \\
\hline $\begin{array}{l}\text { Delimitación de funcio- } \\
\text { nes }\end{array}$ & $\begin{array}{l}\text { Orgánico, estructural y funcional } \\
\text { para el emprendimiento y gestión }\end{array}$ \\
\hline
\end{tabular}

Fuente: Universidad UTE-Proyecto de vinculación con la Sociedad parroquia de Cangahua

\section{Componente 4}

El componente 4 consistió en el desarrollo de capacidades locales que les permitan a los participantes mejorar la prestación de los servicios turísticos y de hospitalidad y como se indicó antes, estas tuvieron un enfoque rural y sobre todo direccionadas hacia la economía popular y solidaria que tiene un considerable apoyo por parte de las entidades públicas para la gestión de recursos.

La certificación que recibieron las personas fue en Gestión del Turismo y Hospitalidad en territorios rurales en donde se dictaron varios temas que son necesarios dentro de la operación del turismo y de la hospitalidad. Este intercambio de conocimiento se lo realizó tanto en el territorio como en las instalaciones de la Universidad UTE, lo que conlleva un aprendizaje colaborativo y experiencial.

Tabla 5 Capacitaciones priorizadas

\begin{tabular}{|c|c|c|c|c|c|}
\hline Certificado en: & Tema & $\begin{array}{c}\text { Horas } \\
\text { docencia }\end{array}$ & $\begin{array}{l}\text { Horas } \\
\text { Autónomas }\end{array}$ & Beneficiarios & Lugar \\
\hline \multirow{10}{*}{$\begin{array}{l}\text { Gestión del } \\
\text { turismo y } \\
\text { hospitalidad } \\
\text { en espacios } \\
\text { rurales }\end{array}$} & $\begin{array}{l}\text { Introducción al turis- } \\
\text { mo comunitario. }\end{array}$ & 1 & 1 & 50 & Cangahua \\
\hline & $\begin{array}{l}\text { Introducción al turis- } \\
\text { mo y a la hospitali- } \\
\text { dad }\end{array}$ & 2 & 2 & 50 & Cangahua \\
\hline & Guianza turística & 3 & 6 & 45 & Cangahua \\
\hline & $\begin{array}{l}\text { Buenas prácticas de } \\
\text { manufactura (BPM) } \\
\text { aplicadas }\end{array}$ & 4 & & 45 & Quito \\
\hline & $\begin{array}{l}\text { Operación en el área } \\
\text { de alojamiento }\end{array}$ & 2 & & 45 & Quito \\
\hline & Cocina ecuatoriana & 8 & & 45 & Quito \\
\hline & $\begin{array}{l}\text { Costos básicos en tu- } \\
\text { rismo y hospitalidad }\end{array}$ & 4 & 2 & 40 & Cangahua \\
\hline & $\begin{array}{l}\text { Comercialización, } \\
\text { mercadeo y } \\
\text { marketing en turismo } \\
\text { y hospitalidad }\end{array}$ & 4 & 1 & 40 & Cangahua \\
\hline & $\begin{array}{l}\text { Emprendimiento } \\
\text { Marco Legal- } \\
\text { Economía Popular y } \\
\text { Solidaria }\end{array}$ & 2 & & 40 & Cangahua \\
\hline & $\begin{array}{l}\text { Atención al cliente } \\
\end{array}$ & 2 & 2 & 40 & Cangahua \\
\hline \multicolumn{3}{|c|}{ Total horas } & 32 & 14 & \\
\hline
\end{tabular}

Fuente: Universidad UTE-Proyecto de vinculación con la Sociedad parroquia de Cangahua

\section{Conclusiones}

La vinculación es un eje sustantivo de la educación superior que aporta al desarrollo local a través de la implementación de proyectos multidisciplinarios que tratan de desarrollar las capacidades locales a través de la participación activa de la comunidad universitaria: -docentes, estudiantes- y las comunidades locales.
Los recursos y atractivos tienen una gran potencialidad turística -estos se manifiestan desde la cultura indígena de los cayambis, los vestigios arqueológicos de Chumillos Central, el paisaje, la crianza de venados, actividades agrícolas y ganaderas, entre otros- y no son aprovechados por los actores locales para la dinamización económica a través de actividades complementarias como es el turismo, y así mejorar la calidad de vida.

Los servicios de alojamiento guardan una básica calidad en el servicio, se han identificado hoteles cercanos y un área de camping, los cuales con el desarrollo de capacidades locales logren posicionar dentro de los servicios turísticos en el territorio.

Los servicios de alimentación que se encuentran cerca de la carrera guardan calidad en el servicio y en la presentación de los platos. Sin embargo, la restauración en los espacios rurales recién se está iniciando por lo que no guarda los lineamientos de calidad básicos ni tampoco la manipulación higiénica de los alimentos.

Debido al carácter rural del territorio, la asociatividad entre los actores jugó un papel importante dentro de las convocatorias para reuniones, validaciones, desarrollo de capacidades locales, intercambio de experiencias, capacitaciones técnicas in situ.

Los productos entregables son el esfuerzo entre la articulación de los estudiantes y docentes con la participación activa de la comunidad local dentro de la planificación de las directrices de vinculación.

La participación multidisciplinaria entre Facultades de la Universidad UTE hace que el alcance del proyecto sea mayor, la Facultad de Hospitalidad y Servicios desde el enfoque turístico, gastronómico y hotelero, la Facultad de Comunicación Artes y Humanidades, diseño gráfico, periodismo y relaciones públicas y la Facultad de Arquitectura y Urbanismo como en el diseño de infraestructura para los servicios turísticos.

\section{Recomendaciones}

Las IES deben trabajar en conjunto para el uso eficiente y eficaz del talento humano, esto a consecuencia que en el territorio de intervención se pudo constatar la participación de varias universidades tanto de la misma oferta académica como en otras áreas productivas.

Continuar con el proyecto de acuerdo a las directrices y productos entregados, entre los cuales se encuentran imagen de marca, rutas turísticas, diseño de proyectos de infraestructura turística, manuales de capacitación interactivos, planes de mejoramiento de emprendimientos, entre otros.

Continuar la gestión por parte de los emprendimientos identificados y desarrollados para la consecución de los objetivos de sus organizaciones; y fortalecer su tejido social para que al momento de participar en proyectos en el territorio se pueda avanzar y conseguir resultados a corto plazo. 


\section{MODELO DE GESTIÓN DE VINCULACIÓN}

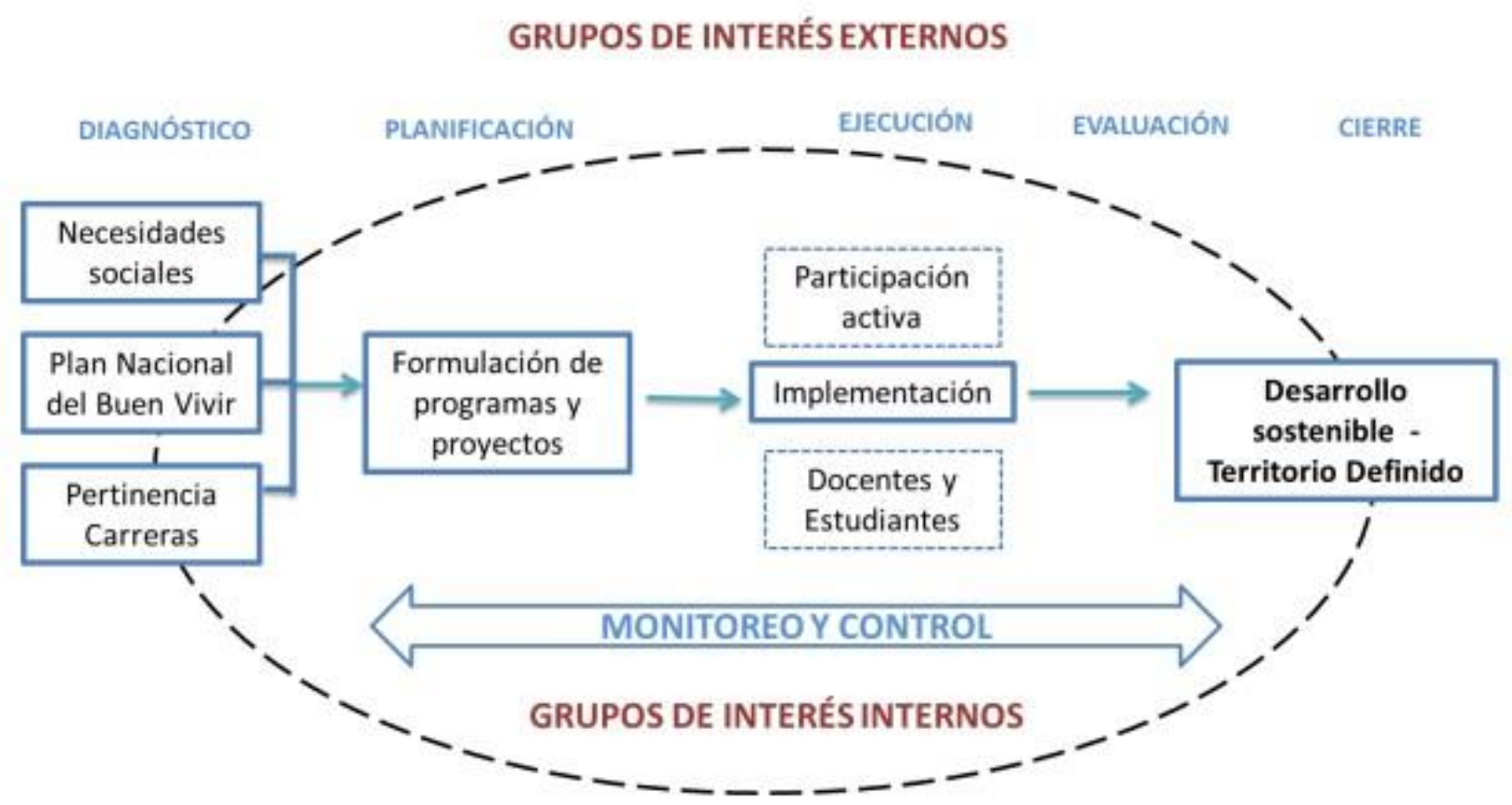

El modelo de gestión de Vinculación de la Universidad UTE debe de alimentarse de modo constante de las experiencias en los proyectos que se vienen ejecutando, con la finalidad de optimizar mecanismos de gestión y de verificación de resultados.

\section{Referencias Bibliográficas}

De Aparicio, X., Chininin Macanchi, M. A., y Toledo Rodríguez, O. C. (2017). El rol de la vinculación en la integración de las funciones sustantivas de la universidad metropolitana del ecuador. Universidad y Sociedad, 9(4), 37-43. Descargado de http: // rus.ucf.edu.cu/index.php/rus

Gobierno Autónomo Descentralizado de Cangahua. (2012). Plan de desarrollo y ordenamiento territorial 20122025.

Instituto Nacional de Estadísticas y Censos. (2010). Censo poblacional.

Orozco, J., y Andrea, A. (2011). Enfoque de gestión universitaria en la vinculación educativa comunitaria. Revista Ciencia UNEMI, 88-96. (ISSN: 1390-4272)

Polaino, C., y Antonio, R. (2017). Vinculación con la sociedad en la universidad de otavalo. Descargado de http://www.redalyc.org/pdf/3735/

373551306004 .pdf? fbclid=IwAR1

-YEZLt 4GOSPWDBIY JqUGGybAeQyqd6y

-AOkskn0m88GNR06j1H7AL-6E

Saltos Briones, G., Odriozola Guitrat, S., y Ortiz Torres, M. (2018). La vinculación universidad-empresagobierno: Una visión histórica y conceptual. Revista ECA Sinergía. (eISSN2528-7869)

Recibido: 3 de mayo de 2019

Aceptado: 18 de junio de 2019 